\title{
Prevalence of syphilis in people living with HIV/AIDS: A Hospital based Cross-Sectional Study
}

\author{
Suresh Jaiswal, ${ }^{1}$ Laxman Banstola, ${ }^{2}$ Manisha Shrestha, ${ }^{1}$ Srijana Sapkota, ${ }^{1}$ Sujan Sharma, ${ }^{1}$ Sunita Lamsal, ${ }^{1}$ Urusha \\ Maharjan, ${ }^{1}$ Sabina Rana Bhujel, ${ }^{1}$ Bishnu Raj Tiwari ${ }^{1}$ \\ ${ }^{1}$ School of Health and Allied Sciences, Faculty of Health Sciences, Pokhara University, Kaski, Nepal. \\ ${ }^{2}$ Department of Pathology, Pokhara Academy of health Sciences, Kaski, Nepal.
}

\begin{abstract}
Introduction: Syphilis, a sexually transmitted disease is one of the oldest diseases caused by the bacterium Treponema pallidum, has been a major public health concern worldwide. The aim is to find out the prevalence of syphilis and its risk factors among people with HIV at Western Regional hospital in Nepal.

Methods: A hospital-based cross-sectional study was conducted at one of the largest public hospitals in Western Region of Nepal. A consecutive 90 HIV-positive patients were recruited prospectively from December 2016 to February 2017. Blood samples and data on sociodemographic and risk factors were collected. Serum were diagnosed for syphilis using Rapid Plasma Reagin (RPR) test.

Results: The prevalence of syphilis infection was $12.2 \%$ in HIV positive participants. Syphilis occurred exclusively in male 7 comparatively 4 in female. Seropositivity of syphilis was seen higher in married than unmarried HIV infected individuals. Syphilis prevalence seems to increase with increasing age, with the highest rate in the age group 3550 years. A decreasing rate of syphilis was observed with increasing educational level, where illiterate HIV-positive participants had higher infection compared with those having at least a certificate.

Conclusion: Higher prevalence of syphilis in people with HIV infection demands the need to target this people to prevent the transmission of both infections. Testing for all HIV-infected people for syphilis and management of the infected would have clinical and epidemiological importance.
\end{abstract}

Keywords: Syphilis, Prevalence, HIV/AIDS, RPR, awareness, health education

\section{INTRODUCTION}

Syphilis, caused by the bacterium Treponema pallidum is one of the oldest diseases and has been a major public health concern worldwide. Nearly 12 million people globally have been infected; of which, almost two-thirds are present at sub-Saharan Africa and Southeast Asia. ${ }^{1}$ Transmission of syphilis is mainly due to unprotected sex and vertical transmission from infected mother to the child. ${ }^{1,2}$ Risk of transmission through blood transfusion is negligible in contexts that ensure improved donor selection and testing as well as refrigerated blood components are transfused. ${ }^{3}$ The possibility of parenteral transmission of syphilis among drug-using individual behaviors has been also reported. ${ }^{4}$ The global efforts to control syphilis mostly focus on screening, treatment and management of pregnant women. However, this strategy has difficulty to be implemented in resource limited countries where access to health services are very difficult. Lack of preventive measures for syphilis in turn makes those areas as a breeding ground and leaves the infection to grow significant public and clinical impacts. ${ }^{5}$
HIV and Syphilis both are sexually transmitted disease, so that a substantial number of people are infected with these agents. HIV has several effects on the presentation, diagnosis, disease progression, and therapy of syphilis. ${ }^{6}$ Syphilis may increase the risk of HIV transmission and acquisition by causing genital ulcers. ${ }^{7}$ Genital ulcer disease is associated to increase risk of HIV infection and is most commonly due to herpes simplex virus (HSV) in HIV positive and negative patients both. ${ }^{8}$ Syphilis continues to have an effect on large numbers throughout much of the world and the past five years has seen a number of outbreaks. HIV makes it more likely for syphilis to present with non-typical features. ${ }^{9}$ Thus, it is important for medical practitioners to be conscious of how syphilis may present in patients with primary HIV infection and its implications for cure and follow-up. Argument has surrounded the area of HIV

Correspondence: Suresh Jaiswal, Lecturer, School of Health and Allied Sciences, Faculty of Health Sciences, Pokhara University, Kaski, Nepal.Email: suuress@gmail.com 
and syphilis co-infection. Some have argued that syphilis has a dissimilar clinical presentation and is more aggressive in people with HIV; others advocate there is little difference.

The present study is therefore, aimed to determine the prevalence and risk factors of syphilis among HIV-infected people in Pokhara valley so that the need to provide regular screening of syphilis screening in HIV- infected population would be discussed.

\section{METHODS}

A cross-sectional study was carried out at Western Regional Hospital, Kaski, Nepal from December 2016 to February 2017. The hospital is among the largest public hospitals in Kaski and provides HIV voluntary counseling, testing and ART as a routine service. Clients who are tested HIV positive are registered at the antiretroviral therapy (ART) clinic and assessed for their disease status. HIV-infected patients are not routinely screened for syphilis and only those with clinical indications are tested. Consecutive HIV-infected individuals with ART status, and who had immunological and biochemical testing were recruited.

In total, $90 \mathrm{HIV}$ positive clients were considered for analysis. In HIV sero-group, participants those who were on syphilis treatment were excluded, as reactive non-Treponema test result may not remain after treatment.

Counsellor interviewed the study participants using structured questionnaire on sociodemographic and other risk factors such as previous history of blood transfusion, insecure injection, multiple sex partners, STIs, and syphilis family history. Blood samples were collected for screening of syphilis using the non-Treponema serologic test, rapid plasma reagin (RPR) test. Laboratory testing was carried out according to the directions of the manufacturers and all tests were run against the positive and negative controls.

Participation was entirely voluntary, and written consent was obtained from the study participants. Any information obtained during the course of study was kept with maximum confidentiality. Syphilis screening was performed at no cost, and those tested positive were reported for further counseling.

Data entry and analysis was performed using SPSS V.16. Results were summarized using descriptive statistics. Pearson's $\chi^{2}$ test was used to evaluate the association.

\section{RESULTS}

Of 99 HIV-positive individuals approached during the study period, 9 were excluded owing to refusal to participate, and in sufficient serum sample and incomplete questionnaire, respectively. Thus, $90 \mathrm{HIV}$ positive were considered for analysis. Majority of HIV-infected participants were male 46(51.1\%) and female 44 (48.9\%). While $79(87.8 \%)$ were married and $11(12.2 \%)$ were unmarried. HIV-infected respondents had age between 5 to 65 years as shown in table 1 .

The prevalence of syphilis infection was $12.2 \%$ in HIV positive participants as shown in fig. 1. Syphilis occurred exclusively in male 7 comparatively 4 in female. Seropositivity of syphilis was seen higher in married than unmarried HIV infected individuals. Syphilis prevalence seems to increase with increasing age, with the highest rate in the age group 35-50 years. A decreasing rate of syphilis was observed with increasing educational level, where illiterate HIV-positive participants had higher infection compared with those having at least a certificate. Similarly, the mode of transmission of HIV was higher by sexual contact as shown in table 2 .

Table 1: Socio-demography of HIV infected individuals

\begin{tabular}{clcc}
\hline & Characteristics & Frequency (\%) & Total (n) \\
\hline \multirow{2}{*}{ Sex } & Male & $46(51.1 \%)$ & \\
& Female & $44(48.9 \%)$ & \\
Marital & Married & $79(87.8 \%)$ & \\
status & Unmarried & $11(12.2 \%)$ & $90(100 \%)$ \\
& Pokhara & $37(41.1 \%)$ & \\
Residence & Outside Pokhara & $11(12.2 \%)$ & $90(100 \%)$ \\
& Inside Kaski & & \\
& Others than Kaski & $42(46.7 \%)$ & \\
& $5-20$ & $8(8.9 \%)$ & \\
& $20-35$ & $14(15.6 \%)$ & \\
Age & $35-50$ & $57(63.3 \%)$ &
\end{tabular}

\section{Syphilis}

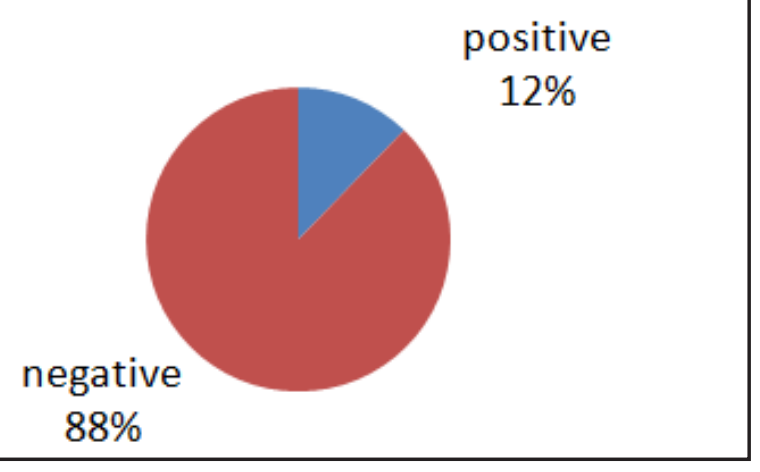

Fig. 1. Showing prevalence of syphilis. 
Table 2: Association of syphilis with the variables

\begin{tabular}{|c|c|c|c|c|c|}
\hline & \multirow{2}{*}{ Characteristics } & \multicolumn{2}{|c|}{ Syphilis } & \multirow{2}{*}{ Chi-square $\left(\chi^{2}\right)$} & \multirow{2}{*}{ P-value } \\
\hline & & Positive ( $\mathrm{n}=11$ ) & Negative $(\mathrm{n}=79)$ & & \\
\hline \multirow{2}{*}{ Sex } & Male & 7 & 39 & & \\
\hline & Female & 4 & 40 & & $0.287^{*}$ \\
\hline \multirow{3}{*}{ Marital status } & Married & 9 & 70 & & \multirow{3}{*}{$0.402 *$} \\
\hline & Unmarried & 2 & 9 & & \\
\hline & Pokhara & 6 & 31 & & \\
\hline \multirow[t]{3}{*}{ Residence } & Outside Pokhara inside Kaski & 2 & 9 & \multirow{3}{*}{1.92} & \multirow{3}{*}{0.382} \\
\hline & Others than Kaski & 3 & 39 & & \\
\hline & Illiterate & 7 & 28 & & \\
\hline \multirow[t]{3}{*}{ Education } & Secondary & 4 & 50 & \multirow{3}{*}{3.280} & \multirow{2}{*}{0.073} \\
\hline & Literate & 0 & 1 & & \\
\hline & Blood transfusion & 0 & 2 & & \\
\hline \multirow{4}{*}{$\begin{array}{c}\text { Mode of } \\
\text { transmission }\end{array}$} & Sexual transmission & 7 & 56 & \multirow{4}{*}{4.322} & \multirow{4}{*}{0.364} \\
\hline & vertical transmission & 0 & 8 & & \\
\hline & Intravenous drug use & 2 & 4 & & \\
\hline & Unknown & 2 & 9 & & \\
\hline \multirow{3}{*}{$\begin{array}{l}\text { CD4 count } \\
\text { group }\end{array}$} & $<200$ & 2 & 16 & \multirow{3}{*}{1.62} & \multirow{3}{*}{0.922} \\
\hline & $200-400$ & 4 & 24 & & \\
\hline & $>400$ & 5 & 39 & & \\
\hline \multirow{4}{*}{ Age group } & $5-20$ & 0 & 8 & \multirow{4}{*}{4.487} & \multirow{4}{*}{0.213} \\
\hline & $20-35$ & 1 & 13 & & \\
\hline & $35-50$ & 10 & 47 & & \\
\hline & $50-65$ & 0 & 11 & & \\
\hline
\end{tabular}

*Fisher exact test $\mathrm{p}$-value

\section{DISCUSSION}

This study shows that the prevalence of syphilis among HIV positive was $12.2 \%$. However, contrasting our result, the coinfection rate was higher among sexually transmitted disease (STD). The seroprevalence of syphilis was highly affected by gender i.e. male in HIV serogroups, similar to findings of Griemberget al. ${ }^{10}$ reported men had a higher risk of syphilis in people living with HIV compared with women. We also found increasing syphilis prevalence with age among HIV-infected individuals, with the highest rate reported in the age group 35-50 years, followed by the others, though no statistically significant was observed. Moreover, our data showed that illiterate HIVinfected participants were affected by syphilis, which point the significance of education to prevent syphilis transmission.

In several regions, where HIV and syphilis has strong association, and transmission of the former is primarily through heterosexual exposure, people with multiple sexual partners would obviously be at higher risk of contracting syphilis as well. Of course, the significance of such risk behavior to influence syphilis prevalence has been documented, where having more than two sexual partners increased syphilis infection compared with those with no sexual partner. ${ }^{11}$

Finally, this study overlooked the importance of including clinical data, which would have been a good opportunity to describe the clinical presentation of syphilis among HIVinfected patients.

\section{CONCLUSION}

Results of this study indicate higher prevalence of syphilis among HIV-infected people. Thus, intervention measures targeting HIV-infected individuals would have paramount importance to prevent transmission of syphilis as well as HIV. As part of this effort, screening all HIV-infected people for syphilis and managing those infected is critically needed. Further studies using a longitudinal design with stronger statistical power would reliably investigate the possible interaction between HIV and syphilis. 


\section{ACKNOWLEDGMENT}

Authors are grateful to the staff and authority of Western Regional Hospital, Kaski, Nepal. The authors like to give a sincere thanks to all the participants despite of the diseased condition, they decided to participate in the study.

\section{REFERENCES}

1. Lynn WA, Lightman S. Syphilis and HIV: a dangerous combination. The Lancet infectious diseases. 2004 Jul 31;4(7):456-66.

2. Kamb ML, Newman LM, Riley PL, Mark J, Hawkes SJ, Malik T, Broutet N. A road map for the global elimination of congenital syphilis. Obstetrics and gynecology international. $2010 \mathrm{Jul}$ 14;1-6.

3. Gardella C, Marfin AA, Kahn RH, Swint E, Markowitz LE. Persons with early syphilis identified through blood or plasma donation screening in the United States. Journal of Infectious Diseases. 2002 Feb 15;185(4):545-9.

4. Loza O, Patterson TL, Rusch M, Martínez GA, Lozada R, Staines-Orozco H, Magis-Rodríguez C, Strathdee SA. Drug-related behaviors independently associated with syphilis infection among female sex workers in two Mexico-US border cities. Addiction. 2010 Aug 1;105(8):1448-56.
5. Meredith S, Hawkes S, Schmid G, Broutet N. World Health Organization. The global elimination of congenital syphilis: rationale and strategy for action.2007.

6. Golden MR, Marra CM, Holmes KK. Update on syphilis: resurgence of an old problem. Jama. $2003 \mathrm{Sep}$ 17;290(11):1510-4.

7. Arora PN, Sastry CV. HIV infection and genital ulcer disease. Indian J Sex Transm Dis 1992; 13: 71-73.

8. Bruisten SM, Cairo I, Fennema H, Pijl A, Buimer M, Peerbooms PG, Van Dyck E, Meijer A, Ossewaarde JM, Van Doornum GJ. Diagnosing genital ulcer disease in a clinic for sexually transmitted diseases in Amsterdam, The Netherlands. Journal of clinical microbiology. 2001 Feb 1;39(2):601-5.

9. Kinghorn GR. Syphilis. In: Cohen J, Powderly WG, eds. Infectious diseases, 2nd edn. London: Mosby, 2004: 807-16.

10. Griemberg G, Bautista CT, Pizzimenti MC, et al. High prevalence of syphilis-HIV co-infection at four hospitals of the city of Buenos Aires, Argentina. Rev Argent Microbiol 2006;38:134-6.

11. Ministry of Health, Disease Prevention and Control Department. AIDS in Ethiopia. Technical document for the fifth report. Addis Ababa: 2004. 